\title{
SELBSTAKUPUNKTUR AUS WISSENSCHAFTLICHER SICHT
}

\section{Self-administered Acupuncture from the Scientific View}

\section{Zusammenfassung}

Die Selbstakupunktur wird seit vielen Jahren nicht nur in China angewendet, die wissenschaftlichen Untersuchungen dazu sind aber sehr spärlich. Es gibt Hinweise, vor allem in chinesischer Sprache, dass eine Selbstakupunktur mit Nadeln durchaus praktikabel möglich ist und auch im Bereich der Lehre dort selbstverständlich angewendet wird. Zur Behandlung einzelner Krankheiten existieren aber nur sehr wenige relevante wissenschaftliche Arbeiten. Ziel dieser kurzen Übersichtsarbeit ist es, diese Publikationen aus der Sicht der Wissenschaft $\mathrm{zu}$ beleuchten und auch neue Varianten wie beispielsweise die selbstapplizierte Lasermedizin in den Fokus des Interesses zu stellen. Ein wichtiger Punkt ist auch jener, dass die Selbstakupunktur mit Nadeln selbstverständlich immer nur nach den gültigen Gesetzen des jeweiligen Landes erfolgen kann und darf. Die Fragestellung, wie man Tradition und Innovation auf dem Gebiet der Selbstakupunktur effizient kombinieren kann, wird auch hier eine der Herausforderungen für die Entwicklung der Akupunktur in der heutigen modernen Gesellschaft sein.

\section{Schlüsselwörter}

Selbstakupunktur, Selbstnadelung, Akupunktur, Akupressur, Laserakupunktur, Traditionelle Chinesische Medizin

\section{Summary}

Self-administered acupuncture has not only been used in China for many years, but the scientific studies on it are very seldom. There are indications, especially in Chinese, that self-acupuncture with needles is quite practicable and is of course also used in teaching there. However, there are only very few relevant scientific papers on the treatment of individual diseases. The aim of this short review article is to shed light on these publications from the perspective of science and also to put new variants such as self-applied laser medicine in the focus of interest. Another important point is that self-acupuncture with needles can of course only be carried out in accordance with the applicable laws of the respective country. The question of how to efficiently combine tradition and innovation in the field of self-administered acupuncture will also be one of the challenges for the development of acupuncture in today's modern society.

\section{Keywords}

self-administered acupuncture, self-needling, acupuncture, acupressure, laser acupuncture, Traditional Chinese Medicine

\section{Einleitung}

„In China sind nur Ärzt $\star^{\star}$ innen oder Medizinstudent ${ }^{\star}$ innen qualifiziert, Selbstakupunktur mit Nadeln durchzuführen. Für Patient ${ }^{\star}$ innen ohne medizinische Grundkenntnisse ist es fast unmöglich, eine Selbstakupunktur zu realisieren und diese wird daher nur von erfahrenen Akupunkteur ${ }^{\star}$ innen praktiziert", berichtet die Professorin Fengxia Liang von

der Hubei Universität für Chinesische Medizin in Wuhan dem Autor des vorliegenden Artikels am 29. Juli 2021. Selbstakupunktur (Abb. 1) bedeutet, sichere und praktikable Akupunkturpunkte am eigenen Körper zu wählen und professionelle Methoden der Akupunktur anzuwenden [1]. Die traditionelle chinesische Akupunktur und Moxibustion sind reich an Konnotationen, die sich stark von der westli- 


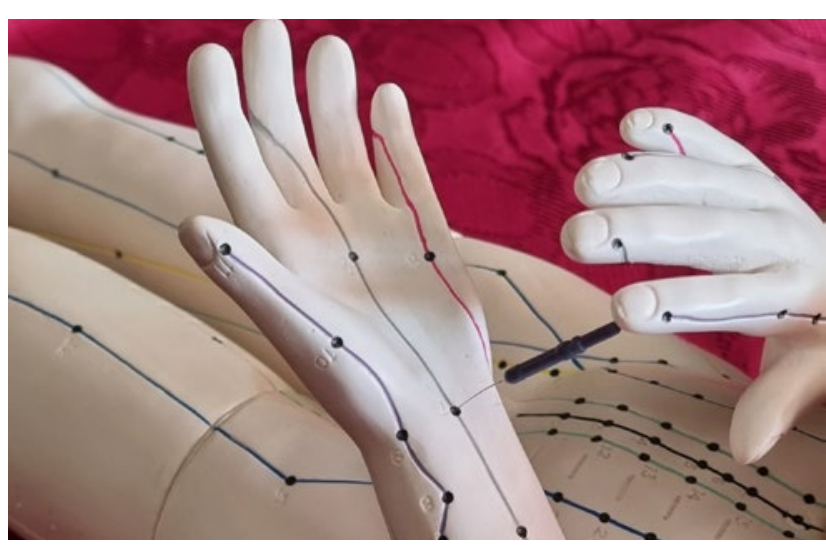

Abb. 1: Manuelle Selbstakupunktur mit Nadeln

\begin{tabular}{|l|c|c|c|}
\hline \multicolumn{4}{|l|}{ Tab. 1: Ergebnisse verschiedener Datenbanken. } \\
\hline $\begin{array}{l}\text { Datenbank/Schlüssel- } \\
\text { worte }\end{array}$ & PubMed & $\begin{array}{l}\text { Google } \\
\text { Scholar }\end{array}$ & CNKI \\
\hline $\begin{array}{l}\text { self-administered } \\
\text { acupuncture }\end{array}$ & 3 & 31 & 9 \\
\hline $\begin{array}{l}\text { self-administered } \\
\text { acupressure }\end{array}$ & 33 & 487 & 20 \\
\hline $\begin{array}{l}\text { self-administered laser } \\
\text { acupuncture }\end{array}$ & 1 & 0 & 0 \\
\hline
\end{tabular}

chen Akupunktur und Moxibustion unterscheiden. Ziel ist es, das Leiden der Patient ${ }^{\star}$ innen zu lindern [2]. In der traditionellen chinesischen Akupunktur kann die heilende Wirkung der Nadeln nur dann optimal sein, wenn die Methode der^des Ärztin ${ }^{\star}$ Arztes, die Wahl der Nadeln, das Gefühl der Patient ${ }^{\star}$ innen und der Unterschied in der körperlichen Fitness perfekt aufeinander abgestimmt sind [3].

Durch eine gut erlernte Selbstakupunkturpraxis durch Ärzt $\star^{\star}$ innen können häufig Schmerzen reduziert werden [3]. Der ${ }^{\star}$ Die Selbstbehandler ${ }^{\star}$ in muss sich die Akupunkturpunktpositionen merken und verschiedene Nadeleinstichrichtungen,Akupunkturtiefen und Stimulationsintensitäten ausprobieren, um die entsprechenden Qi-Empfindungen zu erhalten und zu optimieren [4].Als Akupunkturpatient ${ }^{\star}$ in kann man das innere Qi-Empfinden erfahren [5,6] und manchmal gelingt es gut, sich selbst zu behandeln [7]. Durch eine angelernte Selbstakupunkturpraxis können Ärzt ${ }^{\star}$ innen nicht nur die praxisbezogenen Fähigkeiten verbessern, sondern es gelingt dadurch auch oft, bessere Akupunkturmethoden für Patient $\star_{i n n e n}$ auszuwählen. Einige

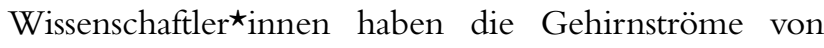
Akupunkteur`innen und Patient ${ }^{\star}$ innen während des Behandlungsprozesses beobachtet und untersucht [8]. Die Er- gebnisse zeigen, dass die elektrische Hirnaktivitäten der Proband $\star_{\text {innen }}$ aufgrund unterschiedlicher Akupunkturtechniken (Sedierung, Tonisierung) unterschiedliche Muster aufweisen und bei der Akupunktur gelegentlich Deltaähnliche Aktivitäten in den Gehirnwellen bei demder Akupunkteur ${ }^{\star}$ in beobachtet werden können. Diese Aktivitäten werden teilweise als Manifestation der Konzentration des Bewusstseins interpretiert. Darüber hinaus ist es interessant, dass sich die Gehirnwellen der Patient ${ }^{\star}$ innen und der Akupunkteur ${ }^{\star}$ innen oft in gleicher Phase befinden. Es konnte festgestellt werden, dass es zu Synchronisierungsmechanismen zwischen Akupunkteur $\star_{i n n e n}$ und Patient $\star^{\star}$ innen kommen kann. Diese Feststellung ist natürlich auch für die Selbstakupunktur von großem Interesse [8].

Im Westen wird die Selbstakupunktur von Patient $\star^{\text {innen }}$ - wenn überhaupt - manchmal als Ersatz- oder Erhaltungstherapie im Spätstadium chronischer Erkrankungen eingesetzt. Im Vergleich zur Akupressur ist die manuelle Akupunktur eine durch den Nadelstich bedingte invasive Behandlung. Die Selbstakupunktur einer ${ }^{\star}$ eines Patientin^Patienten muss unbedingt unter Anleitung pro-

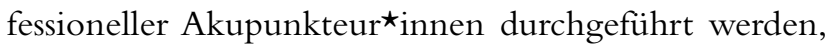
andernfalls ist sie aufgrund einer unsachgemäßen Methode äußerst anfällig für unerwünschte Nebenereignisse.

Dieser Artikel fasst in einem kurzen Überblick erstmals wissenschaftliche Erkenntnisse zum Thema Selbstakupunktur zusammen. Daraus resultierend könnten wissenschaftlich neue Wege und zusätzliche Therapieoptionen für die Patient ${ }^{\star}$ innen entstehen.

\section{Methoden}

\subsection{Suchstrategie}

Die Datenbanken von PubMed, Google Scholar und China National Knowledge Infrastructure (CNKI) wurden bis Anfang August 2021 durchsucht. Die bei der Suche verwendeten Schlüsselwörter waren ,self-administered acupuncture“, ,self-administered acupressure“ und ,selfadministered laser acupuncture". Die Strategie und die Schlüsselwörter wurden entsprechend der jeweiligen Datenbank angepasst. Darüber hinaus wurden zusätzliche Forschungsergebnisse aus chinesischen Arbeiten zum Thema auszugsweise zusammengefasst.

\subsection{Datenbanksuche}

Die Suchanfrage in der PubMed-Datenbank ergab 3 Artikel, die vom Autor näher analysiert wurden (Tab. 1).

Der Schwerpunkt der Analyse lag auf der Interpretation nadelspezifischer Selbstakupunktur. Dabei wurden nur 
Abb. 2: Auswahl von Artikeln für diese Kurzübersicht

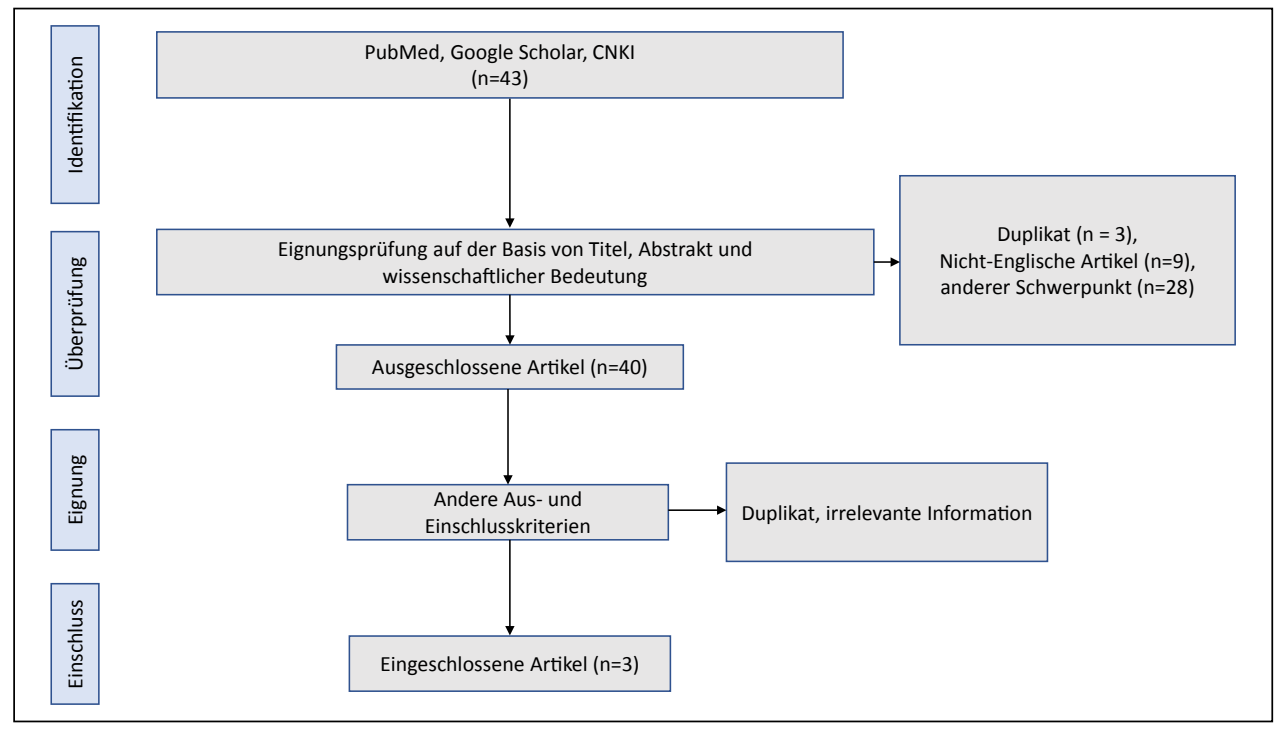

qualitätsgesicherte Forschungsbeiträge zur weiteren Überprüfung/Beschreibung herangezogen (Abb. 2).

\section{Ergebnisse}

\subsection{Selbstakupunktur bei emotionaler Belastung}

Das Ziel einer Machbarkeitsstudie von Davies u. Mitarb. [9] war es, die Akzeptanz, Praktikabilität und Sicherheit des Akupunkturtrainings von Personen zu demonstrieren, die regelmäßig bewusste Selbstnadelungen anwenden, um sich selbst Akupunktur als komplementäre Therapieoption für emotionale Belastungen zu verabreichen. 10 erwachsene Patient ${ }^{\star}$ innen mit der Diagnose einer emotional instabilen Persönlichkeitsstörung, die sich regelmäßig selbst akupunktierten, wurden nach einer Ausgangsbewertung durch einen Psychiater für die Studie rekrutiert. Ein erfahrener Akupunkteur brachte den Teilnehmer ${ }^{\star}$ innen die Selbstakupunktur bei. Während einer 6-wöchigen Intervention hielten die Studienteilnehmer ${ }^{\star}$ innen ihre emotionalen Belastungen, das Bewältigungsverhalten, die Gedanken und die Gefühle in einem Studientagebuch fest. In persönlichen Interviews wurden die Motive der Teilnehmer^innen für die Selbstakupunktur und ihre Erfahrungen mit dieser Methode untersucht. Anhand der Dokumentationen wurde eine Rahmenanalyse durchgeführt, um gemeinsame Themen zu identifizieren. Die Stimmung zu Studienbeginn und nach 6 Wochen wurde mit dem Beck-Depressions-Inventar (BDI) gemessen und Veränderungen im Umgang mit Bewältigungsverhalten und Akupunktur wurden anhand von Studientagebuch-

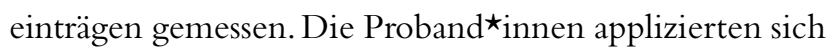
selbst während der 6-wöchigen Intervention regelmäßig
Akupunktur. Die qualitative Analyse identifizierte 2 große Schwerpunkte in Bezug auf den Prozess und die Auswirkungen der Selbstakupunktur. Es gab große Unterschiede in den Auswirkungen, die die Proband $\star_{i n n e n}$ erlebten. Die BDI-Scores zeigten eine signifikante Reduktion von 44,4 auf 34,4 über die 6-wöchige Behandlungsphase. Patient ${ }^{\star}$ innen, die sich absichtliche Selbstverletzung vorstellen konnten, können sicher darin geschult werden, Selbstakupunktur als komplementäre Bewältigungsmöglichkeiten einzusetzen. Die Akzeptanz und Wirksamkeit können je nach den komplexen Motiven, die dem selbstverletzenden Verhalten zugrunde liegen, von Patient ${ }^{\star}$ in zu Patient $\star^{\star}$ in jedoch stark variieren. Während die Pilotstudie darauf abzielte, die generelle Durchführbarkeit einer derartigen Intervention zu untersuchen, deuten die Ergebnisse dieser begrenzten Stichprobe darauf hin, dass die Verwendung von selbst verabreichter Akupunktur die Häufigkeit von selbstverletzendem Verhalten und emotionalen Stress, gemessen mit dem BDI, reduzieren kann [9].

\subsection{Selbstakupunktur in der internen Medizin}

In einer anderen Studie von Walter u. Curtis [10] wird eine 46-jährige Frau mit differenziell diagnostizierter SphinkterOddi-Dysfunktion (SOD) Typ III beschrieben. Nach 2 1/2 Jahren Behandlung der Erkrankung mit einem konventionell-medizinisch-pharmakologischen Ansatz verschlechterten sich die Symptome der Patientin und ergänzende Behandlungsansätze wurden gesucht. Die Patientin begann mit der traditionellen Akupunkturbehandlung, bevor sie von einem Praktiker der westlichen medizinischen Akupunktur zur Selbstverabreichung von Elektroakupunktur ausgebildet wurde. Die Häufigkeit und Intensität schwerer 
nächtlicher Schmerzattacken reduzierten sich und zusätzlich führte die selbst verabreichte manuelle Akupunktur während der Schmerzattacken zu einer schnellen, dauerhaften und vollständigen symptomatischen Schmerzlinderung. Dies war der erste wissenschaftlich veröffentlichte Fallbericht, welcher Elektroakupunktur bei der klinischen Behandlung dieser Erkrankung verwendete. Es zeigt die von der Patientin selbst verabreichte Elektroakupunktur als ein risikoarmes, gut verträgliches Verfahren, das eine wirksame Schmerzlinderung bewirken kann und die Häufigkeit und Schwere von Schmerzattacken reduzierte. Die Autor ${ }^{\star}$ innen dieser Arbeit [10] kommen zum Schluss, dass selbstverabreichte Akupunktur als potenzieller komplementärmedizinischer Ansatz für Patient ${ }^{\star}$ innen mit SOD Typ III in Betracht gezogen werden könnte, bevor auf endoskopische Eingriffe zurückgegriffen wird, welche auch erhebliche Risiken einer Pankreatitis bergen.

\subsection{Gefahren bei Selbstakupunktur}

Bei dieser Zusammenstellung soll die Möglichkeit der Selbstakupunktur aber durchaus auch kritisch betrachtet werden. So kann die Selbstakupunktur auch zu erheblichen Nebenwirkungen und Beschwerden führen. Eine in diesem Jahr erschienene Arbeit von Sung u. Mitarb. [11] zeigt dies eindrucksvoll. Gesichtsabszesse durch eine Candidaalbicans-Infektion sind selbst bei immungeschwächten Patienten eine relativ seltene Erkrankung und es wurden bislang nur sehr wenige Fälle beschrieben. Die Autor ${ }^{\star}$ innen berichten in der Fallstudie von einer Patientin mit multiplen Candida-Abszessen im Gesicht, die durch selbst verabreichte Akupunktur bei einem nicht diagnostizierten Diabetes mellitus verursacht wurden. Die 57-jährige Frau, die sich 2 Wochen zuvor einer Selbstakupunkturbehandlung unterzogen hatte, stellte sich mit einer 1-wöchigen Vorgeschichte von progressiver Schwellung des linken Augenlids, Erythem und Schmerzen vor. Trotz der antibiotischen Behandlung schritt die Läsion fort. Ein chirurgischer Einschnitt und eine Drainage wurden durchgeführt und Candida albicans wurde aus der erhaltenen Eiterkultur isoliert. Bei der Patientin wurde aufgrund eines zufälligen Serumglukosespiegels von $350 \mathrm{mg} / \mathrm{dl}$ und 9,2\% HbA1C Diabetes mellitus vom Typ 2 diagnostiziert. Der Abszess verschwand nach 7 Inzisions- und Drainagezyklen und 4 Wochen intravenöser Fluconazol-Behandlung mit einer geeigneten Kontrolle des Diabetes mellitus [11].

\subsection{Selbstakupunktur in der Onkologie}

Ziel einer weiteren Studie war es, die Wirksamkeit der Selbstakupunktur bei der Behandlung von krebsbedingter
Erschöpfung (cancer related fatigue, CRF) zu beurteilen [12]. Für die Evaluierung wurden Behandlungen durch Therapeut $\star^{\star}$ innen oder durch Selbstakupunktur herangezogen. Untersucht wurden Brustkrebspatientinnen, die an einer randomisierten Studie zur Akupunktur teilnahmen. Das herkömmliche Prozedere wurde um weitere 4 wöchentliche Sitzungen durch erfahrene Akupunkteur ${ }^{\star}$ innen bzw. um 4 selbst verabreichte wöchentliche Akupunktursitzungen (Selbstnadelung) erweitert. Die dritte Gruppe erhielt keine Akupunktur mehr. Bewertet wurden allgemeine Müdigkeit, Stimmung, Lebensqualität und Sicherheit. Insgesamt wurden 197 Patientinnen re-randomisiert, 65 hatten therapeutisch durchgeführte Akupunktursitzungen, 67 hatten Selbstakupunktur/Selbstnadelung und 65 keine weitere Akupunktur. Die primären Ergebniswerte waren zwischen der vom Therapeuten durchgeführten Akupunktur und der Selbstakupunktur gleichwertig. Ein nicht signifikanter Trend zurVerbesserung der Ermüdung wurde am Ende von 4 Wochen in den kombinierten Akupunkturarmen beobachtet. Es gab keine Auswirkungen auf die Stimmung oder Lebensqualität der weiteren Akupunktursitzungen nach 18 Wochen über die in der ersten Studie beobachteteVerbesserung hinaus. Die Autor ${ }^{\star}$ innen der Studie folgern, dass Selbstakupunktur eine akzeptable, machbare und sichere Therapie für Patient ${ }^{\star}$ innen mit CRF sein könnte [12].

\section{Diskussion}

Ergebnisse relevanter Studien zeigten, dass die von der Selbstakupunkturgruppe beobachtete Verbesserung beispielsweise der Fatigue-Scores die gleiche war, wie die von den ausgebildeten Akupunkteur*innen in der Akupunkturgruppe erzielte [12]. Darüber hinaus zeigten Patient $\star_{i n n e n}$ mit vasomotorischen Symptomen nach 6 Jahren Selbstakupunkturbehandlung eine signifikanteVerbesserung [13]. Es ist erwähnenswert, dass eine sichere und wirksame Selbstakupunktur eine professionelle und gründliche Ausbildung der Patient ${ }^{\star}$ innen erfordert oder unter Anleitung von professionellen Akupunkteur ${ }^{\star}$ innen zu erfolgen hat. Ärzt ${ }^{\star}$ innen und auch die Patient ${ }^{\star}$ innen selbst müssen den Gesundheitszustand der zu Behandelnden vollständig einschätzen können, um festzustellen, ob der`die einzelne Patient $\star^{\text {in }}$ die Anforderungen der Selbstakupunktur erfuillt. Wie erwähnt gibt es Berichte, dass nicht diagnostizierte Diabetiker`innen durch falsche Selbstakupunktur infiziert werden können, was im Einzelfall zu multiplen Candida-albicans-Abszessen im Gesicht führen kann [11].

Zusammenfassend lässt sich feststellen, es spielt keine Rolle, ob es sich um eine Selbstakupunktur oder eine 
Akupunktur durch Ärzt ${ }^{\star}$ innen handelt, die wichtigste Prämisse ist, die Sicherheit und Genauigkeit der Akupunkturbehandlung muss gewährleistet sein. Aufgrund der doch erheblichen Unterschiede in der Akupunktur zwischen China und dem Westen gibt es auch Unterschiede in der Akupunktur-Manipulation und der heilenden Wirkung. Stellt man Vergleiche an, so sollten auch die Unterschiede zwischen chinesischen und westlichen kontrollierten randomisierten klinischen Studien in Bezug auf Ausgangssituation, Forschungszweck, Forschungsdesign, Prüfärzt $\star_{\text {in }}$, Akupunkturbehandlungsplan, forschungsmethodische Qualität, Forschungsergebnisse, Forschungsschlussfolgerungen und Forschungsmängel vollständig berücksichtigt werden. Als minimalinvasive Behandlung erfordert die Akupunktur von dem ${ }^{\star}$ der Anwender ${ }^{\star}$ in gute medizinische Kenntnisse und geschickte Techniken. Wenn der ${ }^{\star}$ die Patient ${ }^{\star}$ in nicht über die Voraussetzungen für eine Selbstakupunktur verfügt, kann er andere Formen zur Selbstbehandlung - beispielsweise Akupressurtechniken - erlernen bzw. anwenden.

Eine Rundnadel wird etwa verwendet, um die Körperoberfläche zu massieren und Muskeln zu aktivieren. Damit kann Massage ausgeübt werden und die Akupunkturpunkte können so stimuliert werden, ohne jedoch die Haut zu penetrieren. Dieser Nadeltyp gehört auch zu den historischen 9 Nadeln (Abb. 3) und kann verwendet werden, wie bereits erwähnt, ohne die körperliche Integrität zu verletzen. Die 9 Nadeltypen wurden im Ling Shu, dem zweiten Teil des Gelben Kaisers der inneren Medizin, dem Akupunkturklassiker (Zhen Jing) beschrieben. Gegenwärtig werden diese nicht invasive Akupunkturtherapiemethoden hauptsächlich in der Erholungsphase von zervikaler Spondylose, Gesichtslähmung, Schlaganfall und bei Kopf- und Gesichtssymptomen eingesetzt [14-17]. Darüber hinaus haben einige Wissenschaftler*innen im Rahmen interdisziplinären Projekte traditionelle Rundnadeln mit magnetischen Anwendungen kombiniert, welche dann in der Rehabilitation älterer Menschen und in anderen Bereichen Einsatz finden sollen $[18,19]$.

\section{Selbstakupunktur - Perspektiven}

Akupunktur und Moxibustion gehören zu den Quintessenzen derTraditionellen Chinesischen Medizin. Sie werden von internationalen medizinischen Kreisen aufgrund der evidenzbasierten Wirksamkeit, der Aktualität und des breiten Behandlungsspektrums teilweise anerkannt. Da das Lebenstempo moderner Menschen immer schneller wird, ändern sich auch zahlreiche Methoden der Traditionellen Chinesischen Medizin, um sich der Geschwindigkeit der

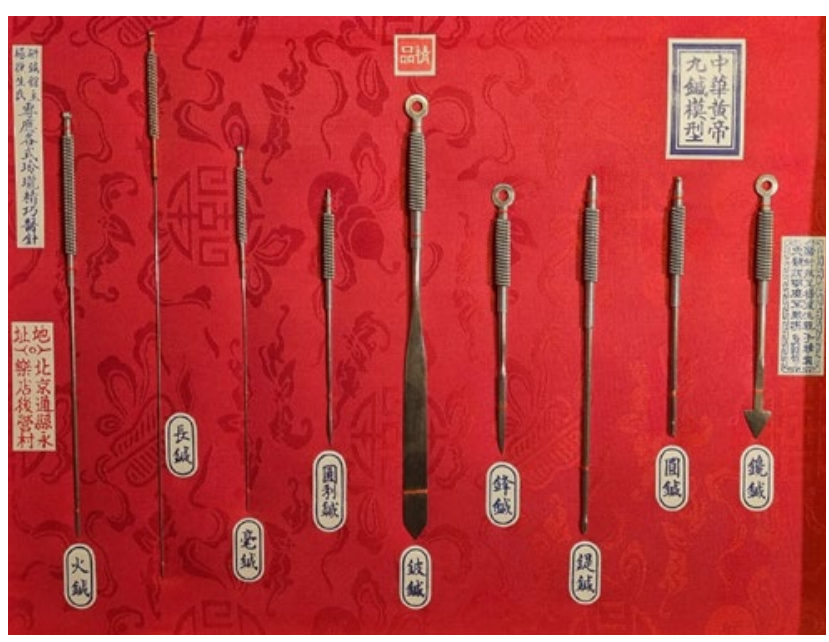

Abb. 3: 9 Nadeltypen, beschrieben im Ling Shu (Foto (C) G. Litscher)

modernen Gesellschaft anzupassen. Die traditionelle Akupunktur und Moxibustion erfordern viel Zeit und Energie. Daher können Selbstakupunktur und physikalische Therapie zu Hause zu einem der Reformtrends der traditionellen Akupunktur werden. Da die Methoden der Akupunktur teilweise streng und kompliziert sind und sicher auch Gefahrenpotenzial beinhalten, müssen selbstverständlich Hygienestandards erfuillt sein. Daher müssen Patient $\star^{\star}$ innen vor der Selbstakupunktur ein standardisiertes Training durchlaufen oder sich von professionellen Akupunkteur^innen anleiten lassen. Sie müssen nicht nur Akupunkturtechniken beherrschen, die Kontraindikationen von Akupunktur und Moxibustion verstehen, sondern auch ein gewisses Erste-Hilfe-Wissen im Umgang mit Notfällen mitbringen. Natürlich hat die Innovation der traditionellen Akupunktur und Moxibustion in Bezug auf neue Materialien und Technologien die Akupunkturbehandlung insgesamt angenehmer und sicherer gemacht.

Eine Möglichkeit in diesem Zusammenhang ist die Laserakupunktur und die damit vergesellschaftete Lasermedizin generell. Tatsächlich gibt es auch erste Arbeiten, wo diese moderne Technik in der Selbstbehandlung angewendet wird. Das Brustkrebs-bedingte Lymphödem ist beispielsweise eine Komplikation mit langfristigen Auswirkungen auf die Lebensqualität nach einer Brustkrebsbehandlung. Sein Management bleibt eine große Herausforderung für die größtenteils weiblichen Patientinnen und das medizinische Fachpersonal. Eine kombinierte physikalische Therapie mit verschiedenen Kombinationen aus Chirurgie, oralen Arzneimitteln, Gewichtsreduktion, mesenchymaler Stammzelltherapie, Kinesio-Tex-Taping, aber auch der Selbstlaserakupunktur könnte bei der Reduzierung von Lymphödemen wirksam sein [20]. Die 
Qualität vieler Originalstudien ist jedoch derzeit noch nicht optimal, sodass in Zukunft randomisierte Kontrollstudien einen hohen Stellenwert haben werden.

Die Fragestellung, wie man Tradition und Innovation auf dem Gebiet der Selbstakupunktur effizient kombinieren kann, wird auch hier eine der Herausforderungen für die Entwicklung der Akupunktur in der heutigen modernen Gesellschaft sein.

\section{Interessenskonflikt}

Der Autor erklärt, dass kein Interessenskonflikt besteht.

\section{Literatur}

[1] Zhang GS, Chang XR, Liu M et al.:Thoughts on the bottleneck and solutions in the teaching of acupuncture practice. Annual Meeting of the Chinese Association of Acupuncture and Moxibustion 2011. Chinesisch.

[2] Gang WJ, Gong CZ, Jing XH:A comparative study of randomized controlled trials of acupuncture in China and the West. Chinese Acupuncture \& Moxibustion 2021;1-13. Chinesisch.

[3] Liu DC, Liu DH: Own experience on acupuncture treatment. Chin J School Doctor 2003; 17: 476. Chinesisch.

[4] Zheng RW:Talking about acupuncture self-testing. Journal of Zhejiang College of TCM 1995; 19: 43. Chinesisch.

[5] Feng CH: My experience of "Qi under the needle". Journal of Zhejiang College of TCM 1998; 22: 36-37. Chinesisch.

[6] Tian CC, Yu L, Murong ZM et al.: Criteria of "arrival of qi" of acupuncture from "reaction of spirit produces curative effect": inheriting Shi Xue-min's thoughts of treating spirit. Zhongguo Zhen Jiu 2021; 41: 666-670. Chinesisch.

[7] Song YZ, Sun HB, Li XL et al.: [Own experience on acupuncture sensation]. Zhongguo Zhen Jiu 2018; 38: 853-856. Chinesisch.

[8] The study of brain waves during acupuncture of the people who treat and being treated. Foreign Medicine (Chinese Medicine Section) 1998; 20: 48. Anmerkung: Keine Autor ${ }^{\star}$ innen gelistet. Chinesisch.

[9] Davies S, Bell D, Irvine F et al.: Self-administered acupuncture as an alternative to deliberate self-harm: a feasibility study. J Pers Disord 2011; 25: 741-754.

[10] WalterWA, Curtis HC: Self-administered electroacupuncture provides symptomatic relief in a patient with sphincter of Oddi dysfunction: a patient's report. Acupunct Med 2013;31:430434 .

[11] Sung JY, Kim JM, Lee JU et al.: Multiple facial candidal abscesses after self-administered acupuncture in a patient with undiagnosed diabetes mellitus: a case report. BMC Complement Med Ther 2021;21: 170.

\section{Danksagung}

Literaturdaten aus China für das vor allem seit der COVID-19-Pandemie äußerst interessante Thema der Selbstbehandlung wurden dem Autor dieser kurzen Übersichtsarbeit dankenswerterweise von Frau Professor Fengxia Liang, Direktorin des Instituts für Akupunktur und Moxibustion an der Hubei Universität für Chinesische Medizin in Wuhan zurVerfügung gestellt.

[12] Molassiotis A, Bardy J, Finnegan-John J et al.: A randomized, controlled trial of acupuncture self-needling as maintenance therapy for cancer-related fatigue after therapist-delivered acupuncture. Ann Oncol 2013; 24: 1645-1652.

[13] Filshie J, Bolton T, Browne D et al.: Acupuncture and self acupuncture for long-term treatment of vasomotor symptoms in cancer patients - audit and treatment algorithm. Acupunct Med 2005; 23: 171-180.

[14] Yang K, Hu GY,Wang YF et al.: Effect of the point-pressing manipulation with spoon needles on the brain-derived nerve growth factors in the cortex of rats with ischemic stroke. Jilin Journal of Chinese Medicine 2021; 41: 701-704. Chinesisch.

[15] Chen YY, Li ZY: Clinical study on cervical spondylosis treated by centro - square needling "Fugui Bao" with spoon needle. Journal of Clinical Acupuncture and Moxibustion 2020; 36 : 39-43. Chinesisch.

[16] Zhang RP: Clinical observation on the treatment of cervical headache with spoon needle combined with routine acupuncture. Shanxi University of Chinese Medicine 2019. Thesis. Chinesisch.

[17] Zhang RP, Wang PR, Xue YN: Clinical observation on the distribution of head tenderness points in patients with cervical headache with spoon needle. Digest of World Latest Medical Information 2019; 19: 213-217. Chinesisch.

[18] Yao JN, Ren WY: Clinical practice research, innovation and application promotion of meridian magnetism therapy with magnetic round needle. Science Herald 2021 (B03). News. Chinesisch.

[19] Yao JN, Cui WG: Clinical observation of 120 cases of insomnia treated by meridian magnetism therapy with magnetic round needle. Science Herald 2021 (B03). News. Chinesisch.

[20] Li L, Yuan L, Chen X et al.: Current treatments for breast cancer-related lymphoedema: a systematic review. Asian Pac J Cancer Prev 2016; 17: 4875-4883.

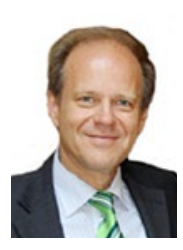

Univ.-Prof. Prof. mult. DI DDr. Gerhard Litscher

Leiter der Forschungseinheit für Biomedizinische Technik in Anästhesie und Intensivmedizin, der Forschungseinheit für Komplementäre und Integrative Lasermedizin

Chairman des TCM Forschungszentrums Graz, Medizinische Universität Graz

Auenbruggerplatz 39,EG19, 8036 Graz, Österreich, E-Mail:gerhard.litscher@medunigraz.at 